\section{Hemolysin sensor}

The ability to identify organic compounds at low concentrations has applications ranging from environmental monitoring to medicine. In a recent issue of Nature (398, 686-690, 1999), Hagan Bayley and his colleagues at Texas A \& M University describe a sensor that can identify individual organic compounds from mixtures of three to four compounds at concentrations as low as 100 picomolar. To construct the sensor, they assembled seven Staphylococcus aureus $\alpha$-hemolysin molecules into a channel-forming pore in a lipid membrane. They then added cyclodextrins-ring-shaped sugars that have an affinity for specific

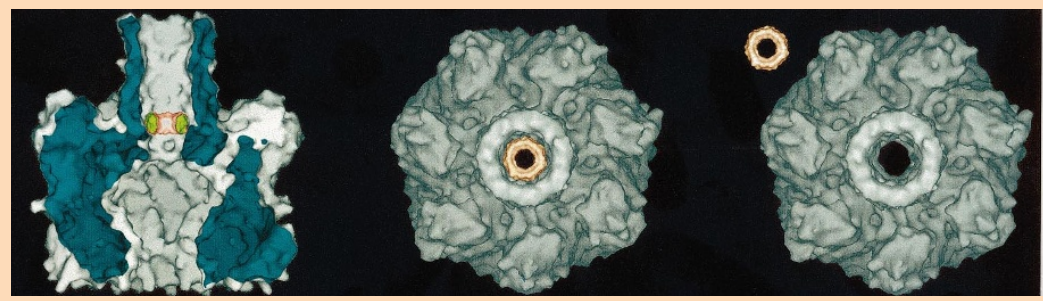

organic molecules - to partially block the pore and narrow its size range. Applying a voltage across the membrane causes a current to flow through the pore, which fluctuates when the relevant organic molecule binds to the cyclodextrin molecule in the pore and blocks it. The frequency of the fluctuations determines its concentration, while the characteristic magnitude and/or length of the fluctuations reveals the molecule's identity. "One could ultimately make arrays of these detectors for different specificities," Bayley suggests. The group is currently working on miniaturizing the sensor to reduce its cost and make it more robust.

\section{Breaking up is hard to do}

A new way to engineer proteins that doesn't rely on sequence homology could provide another means to direct enzyme evolution. Incremental truncation, as it's called, uses combinatorial methods to figure out where a protein can be cut to convert a monomer into a functional heterodimer. It involves chopping up the gene encoding a protein into two nonfunctional pieces with a large area of overlap. After cloning the pieces into separate plasmids and treating with exonuclease III to remove single bases from the overlapping end, the plasmids are religated and transformed into bacteria. The researchers used glycinamide ribonucleotide formyltransferase, an enzyme necessary for de novo synthesis of purines, which allowed selection of transformants in a bacterial strain auxotrophic for purines. As they report in PNAS $(96,3562-3567,1999)$, most of the active heterodimers had breakpoints in loops on the surface of the protein, but surprisingly, some also had breakpoints in the active site. An advantage of this technique over other techniques, such as DNA shuffling, is that it could be used with proteins that only have structural homology, not sequence homology. "This potentially identifies places where you can fuse enzymes and do domain swapping and be successful," says Marc Ostermeier, lead author on the study.

Research News Briefs written by Alka Agrawal and Alan Dove.

\section{Extraterrestrial gene transfer}

Out-of-this-world gene transfer now has a new meaning. On board the NASA space shuttle last October, scientists working on a multi-center project coordinated by the Wisconsin Center for Space Automation and Robotics (WCSAR) have shown that gene transfer into soybean seedlings is 10 -fold more efficent in space than on Earth. Using the Agrobacterium transformation system, they employed green fluorescent protein as a marker to assess gene transfer efficiency. But space-age transformation is not likely to become routine anytime soon. Right now, transferring genes in space"on a regular basis isn't very feasible because of the infrequency of shuttle flights, admits Ray Bula, former director of WCSAR, but the development of the International Space Station will make it easier because it will provide a permanent platform in space. And the cost? "You have to equate [the cost of access to space] to the value of a new variety of plant.

material, and how much an

organization is willing to pay

to have that new variety,"

says Bula. He emphasizes

that this experiment has only been done once, but they have more experiments planned for a shuttle launch in December.

\section{New aptitudes for aptamers}

Using a combination of modular rational design and directed evolution, scientists at Yale University (New Haven, CT) and NexStar (Boulder, CO) have created ribozymes capable of acting as molecular switches in response to a small-molecule ligand. These ribozymes may be useful in devising gene control strategies or biosensors. Taking advantage of the modular nature of enzymes, the researchers combined subdomains with defined activities to generate new enzymes, linking a hammerhead ribozyme to an RNA aptamer that binds flavin mononucleotide (FMN), a small organic molecule (PNAS 96, 3584-3589, 1999). The two modules were joined by a linker sequence of random nucleotides, generating a pool of molecules, which were then subjected to selection for ribozymes either activated or inhibited by FMN. "I expect that almost any ribozyme or deoxyribozyme...will be amenable to [this approach] using almost any aptamer that displays 'adaptive binding' properties," says Ronald Breaker, senior author on the paper. Such enzymes could be activated to either destroy a target mRNA (e.g. as a gene regulatory mechanism) or respond to specific industrial chemicals (e.g., in a biosensor).

\section{A Dam good vaccine?}

In studying the role of DNA adenine methylase (Dam) in Salmonella typhimurium pathogenesis, researchers have developed a strategy that may lead to new vaccines and antibiotics against a variety of bacterial pathogens. Because DNA methylation by Dam is a key gene regulatory mechanism in Salmonella, the team set out to determine whether deletion of the Dam gene would interfere with the normal regulation of genes involved in pathogenesis (Science 284, 967-970, 1999). They found that bacteria lacking Dam constitutively express at least 20 genes that are turned on only during an active infection. These mutants are highly attenuated in a mouse model, but are able to generate protective immunity against wild-type Salmonella. According to the University of California's (Santa Barbara, CA) Michael Mahan, senior author on the study, Dam targeting may be useful in combating a number of diseases: "The Dam gene is present in many pathogens that cause serious health problems worldwide... If Dam acts as a master switch in these organisms, as it does in Salmonella, these findings may lead to a new generation of vaccines and antibiotics." 